AperTO - Archivio Istituzionale Open Access dell'Università di Torino

\title{
Antioxidant and radical scavenging activities of chamazulene
}

\section{This is the author's manuscript}

Original Citation:

Availability:

This version is available http://hdl.handle.net/2318/149892

since 2016-08-09T14:21:00Z

Published version:

DOI:10.1080/14786419.2014.931393

Terms of use:

Open Access

Anyone can freely access the full text of works made available as "Open Access". Works made available under a Creative Commons license can be used according to the terms and conditions of said license. Use of all other works requires consent of the right holder (author or publisher) if not exempted from copyright protection by the applicable law. 
This is the author's final version of the contribution published as:

CAPUZZO A.; OCCHIPINTI A.; MAFFEI M.. Antioxidant and radical scavenging activities of chamazulene. NATURAL PRODUCT RESEARCH. 28 (24) pp: 2321-2323.

DOI: $10.1080 / 14786419.2014 .931393$

The publisher's version is available at:

http://www.tandfonline.com/doi/abs/10.1080/14786419.2014.931393

When citing, please refer to the published version.

Link to this full text:

http://hdl.handle.net/2318/149892 


\title{
Antioxidant and radical scavenging activities of chamazulene
}

\author{
Andrea Capuzzo ${ }^{\mathrm{a}}$, Andrea Occhipinti ${ }^{\mathrm{ab}} \&$ Massimo E. Maffei ${ }^{\mathrm{ab}}{ }^{*}$
}

\begin{abstract}
Essential oils (EOs) of chamomile contain several bioactive compounds, including monoterpenes, sesquiterpenes, triterpenes and fatty acids. Hydrodistillation of chamomile EO induces the formation of chamazulene, a bioactive compound. Chamazulene was isolated from the EO by column chromatography. The total antioxidant capacity confirmed a higher antioxidant activity of chamazulene $\left(\mathrm{IC}_{50}=6.4 \mu \mathrm{g} \mathrm{mL}^{-1}\right)$ than of ascorbic acid $\left(\mathrm{IC}_{50}=12.8 \mu \mathrm{g} \mathrm{mL} \mathrm{mL}^{-1}\right)$, $\alpha$-tocopherol $\left(\mathrm{IC}_{50}=20.5 \mu \mathrm{g} \mathrm{mL}^{-1}\right)$ and of butylated hydroxytoluene $(\mathrm{BHT})\left(\mathrm{IC}_{50}=30.8 \mu \mathrm{g} \mathrm{mL}^{-1}\right)$.

Chamazulene was unable to react with DPPH $\sqrt{ }$. However, when chamazulene was assayed with ABTS $\sqrt{ }$, a strong and significantly $(P<0.05)$ higher free radical scavenging activity was observed $\left(\mathrm{IC}_{50}=3.7 \mu \mathrm{g} \mathrm{mL}^{-1}\right)$, with respect to BHT $\left(\mathrm{IC}_{50}=6.2 \mu \mathrm{g} \mathrm{mL}^{-1}\right)$ and $\alpha$-tocopherol

$\left(\mathrm{IC}_{50}=11.5 \mu \mathrm{g} \mathrm{mL}^{-1}\right)$. The results of this work show that chamazulene is an important factor for the antioxidant power of chamomile oil.
\end{abstract}

\section{Keywords:}

- Matricaria chamomilla L,

- Asteraceae,

- chamazulene,

- $\quad$ antioxidant activity

\section{Introduction}

Bioactive compounds of chamomile (Matricaria spp.) are widely used by the pharmaceutical and cosmetic industries for their antispasmodic, anti-inflammatory and antimicrobial properties and also as a natural hair dye and fragrance (Singh et al. 2011; Ghavimi et al. 2012; Tschiggerl \& Bucar 2012). Among the most important constituents are $\alpha$-bisabolol and bisabolol oxides, spiroethers and $\beta$-farnesene. Another important compound is chamazulene, a bicyclic sesquiterpene blue coloured decomposition product of matricin (prochamazulene), which is formed at high temperatures during steam-distillation processing of essential oils (EOs) (Jakovlev et al. 1983). It has been suggested that chamazulene possessing anti-inflammatory activity (Safayhi et al. 1994) is involved in radical scavenging activity (Rekka et al. 1996) and general antioxidant activity (Sizova 2012). We isolated chamazulene from chamomile EO and tested its antioxidant activity by using DPPH, ABTS, the phosphomolybdenum and the reducing power assays. Here we show that the presence of chamazulene is required for the antioxidant activity of chamomile extracts.

\section{Results and discussion}

Since EOs and plant extracts containing chamazulene have been demonstrated to exert antioxidant and radical scavenging activity (Sizova 2012; Ornano et al. 2013), we isolated chamazulene from chamomile EO by column chromatography (see Supplementary material S1 for materials and 
methods). The content (98\%) and identity of chamazulene were confirmed by GC-FID and GCMS, respectively (Supplementary Figure S1).

Electron-donating capacity reflects the reducing power of bioactive compounds and is associated with their antioxidant activity (Vladimir-Knezevic et al. 2011). Purified chamazulene was then assayed for its reducing power and its activities were compared with the reference antioxidants ascorbic acid, $\alpha$-tocopherol and butylated hydroxytoluene (BHT). Chamazulene showed a higher reducing power $\left(\mathrm{IC}_{50}=7.6 \pm 0.4 \mu \mathrm{g} \mathrm{mL}^{-1}\right)$ than $\alpha$-tocopherol $\left(\mathrm{IC}_{50}=238.9 \pm 3.6 \mu \mathrm{g} \mathrm{mL}^{-1}\right)$ with values closer to butylated hydroxytoluene $(\mathrm{BHT})\left(\mathrm{IC}_{50}=6.5 \pm 0.2 \mu \mathrm{g} \mathrm{mL}^{-1}\right)$ and ascorbic acid $\left(\mathrm{IC}_{50}=3.5 \pm 0.2 \mu \mathrm{g} \mathrm{mL}^{-1}\right)$.

The total antioxidant capacity of chamazulene was assayed by the phosphomolybdenum method and compared with the reference antioxidant compounds. Antioxidant activity of chamazulene $\left(\mathrm{IC}_{50}=6.4 \pm 0.1 \mu \mathrm{g} \mathrm{mL}^{-1}\right)$ was higher than the reference antioxidant compounds: ascorbic acid $\left(\mathrm{IC}_{50}=12.8 \pm 0.1 \mu \mathrm{g} \mathrm{mL}^{-1}\right)$, BHT $\left(\mathrm{IC}_{50}=30.8 \pm 0.3 \mu \mathrm{g} \mathrm{mL}^{-1}\right)$ and $\alpha$-tocopherol $\left(\mathrm{IC}_{50}=20.5 \pm 0.2 \mu \mathrm{g} \mathrm{mL}^{-1}\right)$.

Free radical scavenging activities of extracts were assayed by using the stable DPPH radical. Chamazulene was found to be inactive to DPPH $\sqrt{ }$. This result depends on the ability of chamazulene to interact with the stable, nitrogen-centred DPPH $\sqrt{ }$, which is indicative of its reduced potency in an iron-free system (Rekka et al. 1996). The generation of the ABTS radical cation forms the basis of one of the spectrophotometric methods that have been applied to the measurement of the total antioxidant activity of solutions of pure substances (Re et al. 1999). When chamazulene was assayed with ABTS $\sqrt{ }$, a strong and significantly higher free radical scavenging activity was observed $\left(\mathrm{IC}_{50}=3.7 \pm 0.7 \mu \mathrm{g} \mathrm{mL}^{-1}\right)$ than $\alpha$-tocopherol $\left(\mathrm{IC}_{50}=11.5 \pm 1.1 \mu \mathrm{g} \mathrm{mL}^{-1}\right)$ and with intermediate values between BHT $\left(\mathrm{IC}_{50}=6.2 \pm 0.5 \mu \mathrm{g} \mathrm{mL} \mathrm{m}^{-1}\right)$ and ascorbic acid $\left(\mathrm{IC}_{50}=1.6 \pm 0.6 \mu \mathrm{g} \mathrm{mL}^{-1}\right)$. The observed $\mathrm{IC}_{50}$ values of chamazulene were also comparable to those observed for other strong radical scavenging compounds such as quercetin, delphinidin and lycopene (Re et al. 1999).

The antiradical activity characterises the ability of the compounds to react with free radicals (in a single free radical reaction), whereas the antioxidant activity represents the ability to inhibit the process of oxidation. Consequently, test systems using DPPH and ABTS give information on the radical scavenging or antiradical activity, although in many cases this activity does not correspond to the antioxidant activity (Tirzitis \& Bartosz 2010). Our results are in agreement with recent findings where the $\mathrm{ABTS}^{\sqrt{+}}$-scavenging activity of the chamazulene-rich Artemisia arborescens EO was found to be much higher than its capacity to scavenge the DPPH radical (Ornano et al. 2013).

\section{Conclusions}

The results of this work show that chamazulene is an important contributor to the antioxidant and radical scavenging activity of chamomile EOs. The isolation of chamazulene from chamomile EO allowed evaluating its bioactive properties showing both a strong radical scavenger and a powerful antioxidant ability along with a strong reducing power. Our data confirm the need to avoid DPPH assay to test chamazulene radical scavenging activity owing to its interference with the nitrogencentred DPPH $\sqrt{ }$ and suggest the use of ABTS.

\section{Supplementary material}

Experimental details relating to this article are available online, alongside Figure S1. 


\section{Acknowledgement}

This work was supported by the Doctorate School of Pharmaceutical and Biomolecular Sciences of the University of Turin, Italy.

\section{References}

1. GhavimiH, ShayanfarA, HamedeyazdanS, ShivaA, GarjaniA. 2012. Chamomile: an ancient pain remedy and a modern gout relief - a hypothesis. Afr J Pharm Pharmacol.6:508-511, 10.5897/AJPP10.197.

2. JakovlevV, IsaacO, FlaskampE. 1983. Pharmacological investigations with compounds of chamomile. Planta Med.49:67-73.

3. OrnanoL, VendittiA, BalleroM, SannaC, QuassintiL, BramucciM, LupidiG, PapaF, VittoriS, MaggiF, BiancoA. 2013. Chemopreventive and antioxidant activity of the chamazulene-rich essential oil obtained from Artemisia arborescens L. growing on the Isle of La Maddalena, Sardinia, Italy. Chem Biodivers.10:1464-1474.

4. ReR, PellegriniN, ProteggenteA, PannalaA, YangM, Rice-EvansC. 1999. Antioxidant activity applying an improved ABTS radical cation decolorization assay. Free Radic Biol Med.26:1231-1237.

5. RekkaEA, KourounakisP, KourounakisPN. 1996. Investigation of the effect of chamazulene on lipid peroxidation and free radical processes. Res Commun Mol Pathol Pharmacol.92:361-364.

6. SafayhiH, SabierajJ, SailerER, AmmonHPT. 1994. Chamazulene - an antioxidant-type inhibitor of leukotriene B-4 formation. Planta Med.60:410-413.

7. SinghO, KhanamZ, MisraN, SrivastavaMK. 2011. Chamomile (Matricaria chamomilla L.): an overview. Pharmacogn Rev.5:82-95.

8. SizovaNV. 2012. Composition and antioxidant activity of essential oils containing azulene derivatives. Pharm Chem J. 46:369-371, 10.1007/s11094-012-0800-6.

9. TirzitisG, BartoszG. 2010. Determination of antiradical and antioxidant activity: basic principles and new insights. Acta Biochim Pol. 57:139-142.

10. TschiggerlC, BucarF. 2012. Guaianolides and volatile compounds in chamomile tea. Plant Food Hum Nutr.67:129-135.

11. Vladimir-KnezevicS, BlazekovicB, StefanMB, AlegroA, KoszegiT, PetrikJ. 2011. Antioxidant activities and polyphenolic contents of three selected Micromeria species from Croatia. Molecules. 16:1454-1470. 